\title{
The intermuscular 3-7 Hz drive is not affected by distal proprioceptive input in myoclonus-dystonia
}

\author{
J. N. van der Meer • A. C. Schouten · L. J. Bour • \\ E. de Vlugt $\cdot$ A. F. van Rootselaar $\cdot$ \\ F. C. T. van der Helm • M. A. J. Tijssen
}

Received: 7 July 2009 / Accepted: 19 January 2010 / Published online: 16 February 2010

(C) The Author(s) 2010. This article is published with open access at Springerlink.com

\begin{abstract}
In dystonia, both sensory malfunctioning and an abnormal intermuscular low-frequency drive of 3-7 Hz have been found, although cause and effect are unknown. It is hypothesized that sensory processing is primarily disturbed and induces this drive. Accordingly, experimenter-controlled sensory input should be able to influence the frequency of the drive. In six genetically confirmed myoclonus-dystonia (MD) patients and six matched controls, the low-frequency drive was studied with intermuscular coherence analysis. External perturbations were applied mechanically to the wrist joint in small frequency bands $(0-4,4-8$ and $8-12 \mathrm{~Hz}$; 'angle protocol) and at single frequencies (1, 5, 7 and $9 \mathrm{~Hz}$; 'torque' protocol). The low-frequency drive was found in the neck muscles of $4 \mathrm{MD}$ patients. In these patients, its frequency did not shift due to the perturbation. In the torque protocol, the externally applied frequencies could be detected in all controls and in the two patients without the common drive. The common low-frequency drive was not be affected by external perturbations in MD patients. Furthermore, the torque protocol did not induce intermuscular
\end{abstract}

J. N. van der Meer · L. J. Bour · A. F. van Rootselaar ·

M. A. J. Tijssen

Department of Neurology and Clinical Neurophysiology,

Academic Medical Centre, Amsterdam, The Netherlands

A. C. Schouten - E. de Vlugt - F. C. T. van der Helm

Laboratory for NeuroMuscular Control,

Department of Biomechanical Engineering,

Delft University of Technology, Delft, The Netherlands

M. A. J. Tijssen ( $\square)$

Department of Neurology H2-237, Academic Medical Centre,

University of Amsterdam, PO BOX 22660,

1100 DD Amsterdam, The Netherlands

e-mail: M.A.Tijssen@amc.uva.nl coherences at the applied frequencies in these patients, as was the case in healthy controls and in patients without the drive. This suggests that the dystonic $3-7 \mathrm{~Hz}$ drive is caused by a sensory-independent motor drive and sensory malfunctioning in MD might rather be a consequence than a cause of dystonia.

Keywords EEG $\cdot$ EMG $\cdot$ Dystonia $\cdot$ Proprioception · Sensorimotor integration

\section{Introduction}

Dystonia is characterized by twisting movements and abnormal postures due to involuntary, sustained muscle contractions (Yanagisawa and Goto 1971; Vitek 2002; Tarsy and Simon 2006). It has been frequently hypothesized that sensory malfunction induces or aggravates symptoms associated with dystonia. (Breakefield et al. 2008; Abbruzzese and Berardelli 2003). Functional imaging studies (fMRI, PET) have revealed similar abnormalities in the functioning of the brain. Abnormal homuncular organization and increased activations in the somatosensory cortex have been found for different types of dystonia (Bara-Jimenez et al. 1998; Sanger et al. 2002; Dresel et al. 2006). That dystonia has a sensory origin is furthermore connoted by the observation that some patients can use a 'sensory trick' to alleviate their symptoms. (Naumann et al. 2000). A range of sensory stimuli (e.g. high-frequency cutaneous vibrations, electric jolts) have been applied in cervical dystonia (Leis et al. 1992; Bove et al. 2004) and in writer's cramp and musician's dystonia (McDonnell et al. 2007; Rosenkranz et al. 2008) to attempt to alleviate symptoms.

One specific characteristic of cervical dystonia is the presence of a low-frequency drive, between 3 and $7 \mathrm{~Hz}$, 
which innervates two (or more) muscles, causing them to contract simultaneously (Tijssen et al. 2002; Grosse et al. 2004; Foncke et al. 2007b). Coherence analysis is a frequency analysis method that, when applied to two sets electromyography (EMG) data, can detect this drive (Halliday et al. 1995). So far, this low-frequency drive has been investigated separately from sensory malfunctions (Grosse et al. 2004; Breakefield et al. 2008). Our goal was to see if the sensory responsiveness and the presence of a low-frequency drive in dystonia somehow are related. In case, this relationship exists an externally applied input could alter the drive, driving it to higher or lower frequencies.

Since the origin of dystonia can be from multiple sources, we have measured the low-frequency drive in a homogeneous group of patients with myoclonus-dystonia (MD). MD is an autosomal dominant hereditary form of dystonia characterized by myoclonic jerks and dystonic postures or movements of the upper body. The condition usually becomes clinically manifest within the first two decades of life and is alcohol responsive (Foncke et al. 2006; Gerrits et al. 2006). In MD patients, the low-frequency drive had been reported at 3-7 Hz (Foncke et al. 2007b). In another report, the connection with the basal ganglia was confirmed as a low-frequency drive was also reported between EMG recordings from dystonic muscles and from local field potential (LFP) recordings from intracerebral deep brain stimulation electrodes positioned in the Globus Pallidus internus (GPi) (Foncke et al. 2007a). This drive between LFP and EMG was in an extended frequency range, i.e., 3-15 Hz. Since no cortical abnormalities were found in MD (Li et al. 2008), dystonia in MD is likely due to sub-cortical malfuction.

System analysis gives the opportunity to study whether sensory malfunction is related to the occurrence of a pathological low-frequency drive. This approach has been used to describe spinal feedback control mechanisms for posture and muscle activity of the hand and arm (de Vlugt et al. 2002; van der Helm et al. 2002; Schouten et al. 2008). In dystonia, it could be hypothesized that the low-frequency drive can be explained by disturbed interaction between the sensory and motor parts of a sensory-motor feedback loop (Rosenblum and Pikovsky 2004). Malfunctions in the sensory part in combination with these mutual interactions could cause a resonance, characterized by a motor output to affected muscles with an abnormal frequency between 3 and $7 \mathrm{~Hz}$. This resonance would therefore be responsible for the symptoms associated with dystonia. System analysis predicts that this resonance should be influenced by modifying the total sensory input with experimenter-controlled perturbations. If this extra sensory input is strong and occurs at a frequency slightly higher or lower than the resonance frequency, it would force the resonance to occur at that imposed frequency. In the healthy subject sensory- motor system, external perturbations cause reflexive feedback; i.e., counter-forces that are generated by the reflex system that have the same frequency as the perturbation itself.

In the present study, proprioceptive stimuli were provided using a wrist manipulator (WM) (Schouten et al. 2006) in two protocols. The 'angle' protocol consisted of perturbations in different frequency bands which were designed to stimulate muscle tendon organs only. The 'torque' protocol had perturbations at single frequencies, designed to stimulate muscle tendon organs, Golgi tendon organs and also induce passive movements of the wrist. A shift of the peak frequency of the intramuscular drive in one of these protocols would indicate that in MD, proprioceptive input or processing plays a role in the generation of dystonia.

\section{Methods}

\section{Participants}

Six confirmed, DYT-11 positive MD patients (3 women, mean age 47 years, range 29-65 years) with primary dystonia in the neck and arm muscles were recruited from the neurology out-patient clinic (Table 1). Six age- and sexmatched control subjects (mean age 47 years, age range 29-62 years) were also studied. Control subjects had no known neurological dysfunction and were not receiving medication. The study was in accordance with the Declaration of Helsinki and was approved by the Medical Ethical Committee of the Academic Medical Centre, Amsterdam.

Patients were asked which side they felt to be most affected by dystonia; this was subsequently checked and confirmed against clinical records at the time of the experiment. All subjects were briefed about the protocols and prepared with EMG and EEG electrodes by the experimenters. Control subjects were tested according to the same protocols as the MD patients. Each experiment took approximately 75 min including instruction and pauses to prevent fatigue.

\section{Wrist manipulator}

Perturbations were applied at the wrist joint by means of the WM (Schouten et al. 2006). The WM is a rotating device with a single degree of freedom that is able to exert flexion and extension perturbations around the wrist joint. It is driven by an electromotor attached to a lever and handle. The perturbations consisted of varying angle (position) and varying torque (angular force) as depicted in Fig. 1. Counter-torques applied by the subject were measured by a torque transducer mounted halfway along the lever. The 
Table 1 Characteristics of MD patients

\begin{tabular}{llllllll}
\hline $\begin{array}{l}\text { MD } \\
\text { patient }\end{array}$ & $\begin{array}{l}\text { Sex/age } \\
\text { (year) }\end{array}$ & $\begin{array}{l}\text { Arm } \\
\text { measured }\end{array}$ & $\begin{array}{l}\text { Distribution } \\
\text { of dystonia }\end{array}$ & $\begin{array}{l}\text { Distribution } \\
\text { of myoclonus }\end{array}$ & BFMDRS/UMRS & $\begin{array}{l}\text { Months since } \\
\text { last BTX treatment }\end{array}$ & Medication \\
\hline 1 & M/49 & R & N, T, WC & N, UL & NA $^{\text {a }}$ & 10 & $\begin{array}{c}\text { Trihexyphenidyl, } \\
\text { paroxetine, phenobarbital, } \\
\text { clonazepam } \\
\text { Atenonol }\end{array}$ \\
2 & F/47 & L & N & N, UL & $13 / 9$ & 2 & - \\
3 & F/29 & R & N & N, UL & $17 / 4$ & 2 & Venlafaxine \\
4 & M/49 & L & N, UL & Cr, N, UL, LL & $15 / 20$ & 1 & - \\
5 & M/65 & R & N & - & $8 / 3$ & 20 & - \\
\hline
\end{tabular}

$C r$ cranial, $N$ neck, $T$ trunk, $L L$ lower limbs, $U L$ upper limbs, WC Writer's cramp, BFMDRS Burke Fahn Marsden dystonia rating scale, $U M R S$ Unified myoclonus rating scale, $N A$ not available.

${ }^{a}$ Patient 1 was more affected with dystonia than myoclonus in the neck.. Myoclonus was also present in the trunk.

${ }^{\mathrm{b}}$ In Patient 6, the myoclonus in the neck was more pronounced

arm was strapped to the arm support to prevent arm movement. The angle and torque of the handle were displayed on a computer screen, along with maximum and minimum target values. Participants were seated in a chair and instructed to grasp the handle in their hand, while keeping the deviations as small as possible.

\section{Angle protocol}

Subjects were instructed to counter a constant torque of $1 \mathrm{Nm}$ while angle perturbations were superimposed. In three conditions, angle perturbation frequency bands were set to $0-4,4-8$ and $8-12 \mathrm{~Hz}$ (see Fig. 1). The fourth (isometric) condition did not have perturbations. This condition was included to compare coherence results with other published reports. The angle perturbations had a root-meansquare of about 1 degree of rotation. They were constructed as multi-sine signals with length of 2048 samples (frequency resolution, 0.48) and with equal power at all frequencies within the bandwidth in which they were applied. The multi-sine signals were crested to increase the compactness of the signals (Pintelon et al. 2003). To subjects, the angle perturbations had a 'jittering' sensation. For each condition, 6 trials of 15 segments were recorded, yielding 90 segments of 2,048 s for coherence analysis. Performance in this task is defined as RMS deviation between subjectapplied torque and the constant torque from the WM. The isometric torque of $1 \mathrm{Nm}$ resulted in EMG activity of the FCR comparable with $\sim 15 \%$ of maximum voluntary exertion, and this muscle activity remained constant, regardless of perturbations.

\section{Torque protocol}

Subjects were instructed to resist a constant torque of 0.5 $\mathrm{Nm}$, superimposed with single-frequency sinusoidal torque perturbations with amplitude of $0.2 \mathrm{Nm}$. In four conditions, torque perturbation frequencies were set to $1,5,7$ and $9 \mathrm{~Hz}$ (see Fig. 1). For each condition, 2 trials of 30 consecutive segments were recorded, yielding 60 segments of $1,024 \mathrm{~s}$ of data for coherence analysis. Performance in this task is defined as root mean squared (RMS) deviation from zero angle. The sinusoid torque perturbations resulted in a rotational motion of the wrist that had the same period as the perturbation.

\section{Signal recording}

Surface EMG recordings were performed with $9 \mathrm{~mm}$ diameter $\mathrm{Ag} / \mathrm{AgCl}$ electrodes from six muscles: first dorsal interosseus (FDI), extensor carpi radialis (ECR), flexor carpi radialis (FCR), deltoideus medialis (DEL), splenius capitis (SC) and sternocleido mastoid (SCM). EMG electrodes were placed $2-3 \mathrm{~cm}$ apart on the muscle belly (except for FDI, where one electrode was sited over the metacarpophalangeal joint). Impedances were below $5 \mathrm{kOhm}$. If the most dystonic side was the right-hand side (RHS), all recordings were made from this side of the patient and from the matched control, with the exception of SC where recordings were made from the left-hand side (LHS). This setup was mirrored if the patients most affected side was left.

Although in MD no cortical abnormalities were found, a cortical relay seems essential if basal nuclei are involved in the generation of this common drive, so we also measured EEG. This was performed with $9 \mathrm{~mm}$ diameter $\mathrm{Ag} / \mathrm{AgCl}$ electrodes with impedances below $5 \mathrm{kOhm}$. The EEG apparatus was set up as follows. In patients most affected on the RHS, electrodes were placed to allow for source derivations on the FC3 and CP3 scalp location using 5 electrodes with a distance between topographically adjacent electrodes of $10 \%$ (FC1, C1, CP1, F3, FC3, C3, CP3, CP3, FC5, C5 and 

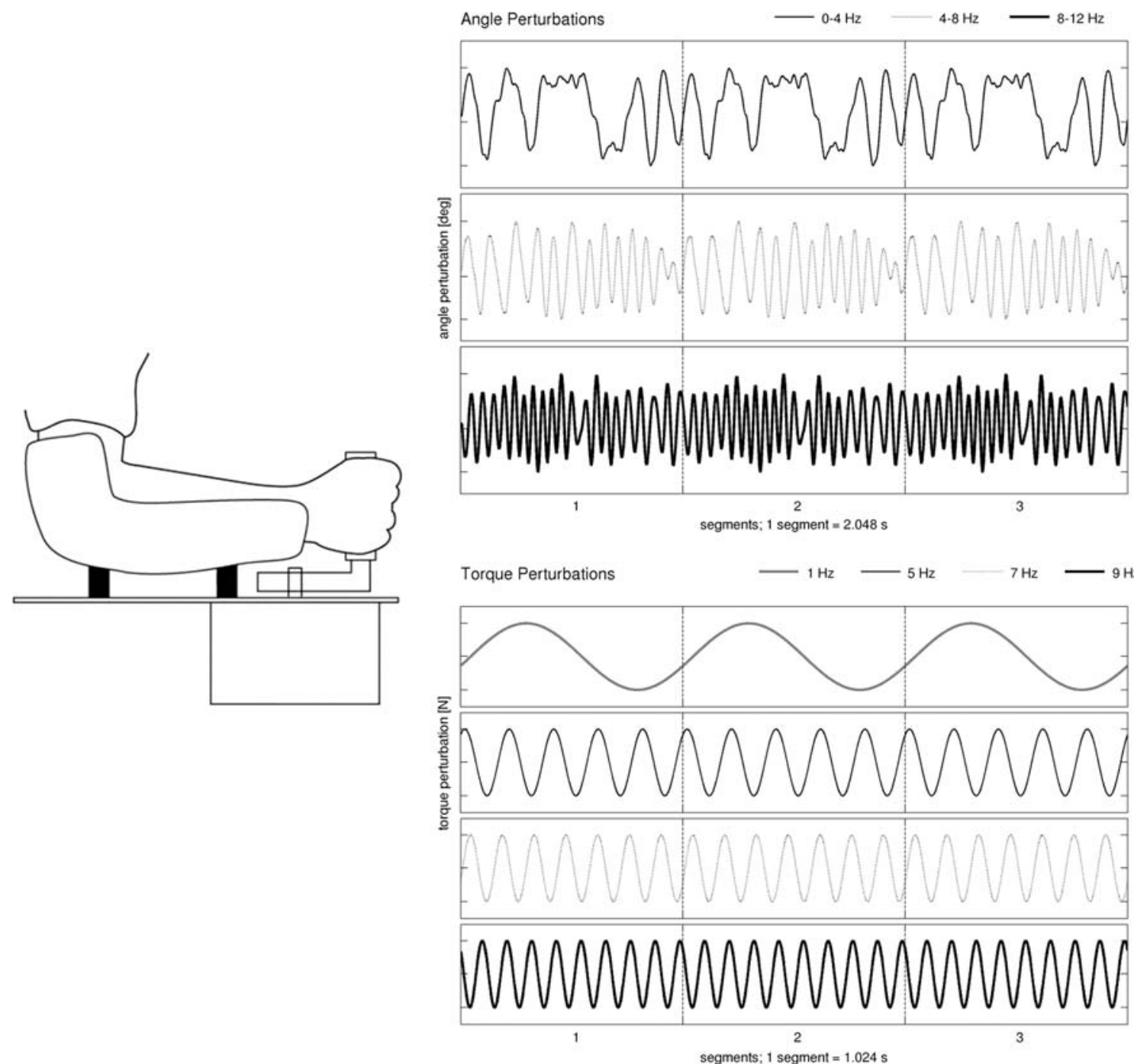

Fig. 1 Wrist manipulator schematic (left) and angle (top-right) and torque (bottom-right) perturbations. Three segments are shown. For the angle perturbations, the signals are constructed in the frequency

CP5, Hjörth derivation). This setup was mirrored around the mid-sagittal plane when the most affected side was the LHS. These contralateral source derivations are denoted as SFC and SCP. Placement of electrodes was identical in patients and their matched controls. In addition to EEG and EMG, the angle and generated by the WM and applied by the subject, as well as the electrocardiogram (ECG), were recorded.

All signals were highpass filtered at $0.005 \mathrm{~Hz}(6 \mathrm{~dB} / \mathrm{oct})$ to prevent drift and lowpass filtered at $350 \mathrm{~Hz}(6 \mathrm{~dB} /$ oct $)$ to prevent aliasing. Signals were monitored continuously online during the experiment, sampled at $1 \mathrm{kHz}$, and stored on the computer using the Schwartzer 34 amplifier system (Schwartzer GmbH, Medical Diagnostic Equipment, Munich, Germany) and Brainlab software (OSG bvba, Rumst, Belgium). domain to have equal power at all perturbation frequencies (from beginning to end in steps of $0.5 \mathrm{~Hz}$ ). For the torque perturbations, there was power at only a single frequency

Data were recorded in trials of $30 \mathrm{~s}$, alternating with rest periods until the subject indicated that the trial could proceed again (usually 30-60 s). In total, 8 trials for the torque protocol ( 2 trials for each condition) and 24 trials for the angle protocol (6 trials for each condition) were recorded.

\section{Coherence}

\section{Preprocessing}

Data were inspected visually and processed off-line using MATLAB 7.3 (The Mathworks Inc, Natick, MA, USA). To remove ECG artifacts in the EMG, a QRS detection algorithm identified heart beat events. Average QRS template were calculated and subtracted for each EMG channel (Bloch 1983; Levine et al. 1986). This was followed by 
high-pass filtering at $30 \mathrm{~Hz}(6 \mathrm{~dB} / \mathrm{oct})$ using a digital second order digital Butterworth filter. EMG data were subsequently rectified to enhance the firing rate information of the signal (Myers et al. 2003). Visual inspection of EEG Hjörth derivations did not show electro-oculogram (EOG), muscle or motion artefacts, therefore no additional correction algorithm or filter was applied to the EEG. EEG and EMG data were subsequently segmented into 60 segments of 1,024 ms data points for the torque protocol and $90 \mathrm{seg}$ ments of 2,048 ms data points for the angle protocol.

\section{Coherence analysis}

Frequency analysis was performed using Matlab and NeuroSpec software (Division of Neuroscience and Biomathematical Systems, University of Glasgow, UK, http:// www.neurospec.org). The Discrete Fourier Transform of the segments of data points was used to contract autospectra for each EEG and EMG segment. Coherence is an extension of Pearson's correlation coefficient and ranges from 0 (no linear association) to 1 (perfect linear association) and is the absolute square of the cross-spectrum, normalized by the product of the absolute autospectra. For the angle protocol, frequency spectral resolution was $0.5 \mathrm{~Hz}$ and for the torque protocol, $1 \mathrm{~Hz}$. Intermuscular coherence (IMC) and corticomuscular coherence (CMC) spectra were estimated in the 1 to $30 \mathrm{~Hz}$ range and formally assessed in the $1-12 \mathrm{~Hz}$ range (the maximum frequency of the perturbations used in this study). Shoulder-neck IMC was estimated between SC and DEL, and 'proximal' neck-neck IMC between SC and SCM. Arm CMC was estimated between FCR and SCP, and Neck CMC between SC and SCP.

\section{Frequency shift and reflexive feedback}

For both protocols, the intermuscular drive in each individual patient was detected when coherence in a band between 3 and $7 \mathrm{~Hz}$ rose above the $95 \%$ significance threshold for three or more consecutive frequency bins. If found, the central "drive" frequency was calculated by averaging all these frequency bins, weighted by the value of the coherence. The central frequency would shift if it would increase and decrease in the same way as the frequency of the perturbations. W applied a second criterion to detect if perturbations cause reflexive feedback. Reflexive feedback due to WM perturbations would generate EMG-EMG coherence at exactly the same frequency as the frequency of the torque perturbation. For the torque protocol, since it used singlefrequency perturbations, reflexive feedback occurred if the coherence value at the same frequency as the perturbation was at least twice as high as the $95 \%$ significance level and the coherence values at the surrounding frequency bins.
The coherence values at the perturbation frequencies were subjected to a two-sample $t$ test to test for differences between patients and controls. Before statistical testing these values were transformed (Fisher Z) to approximate a normal distribution (Rosenberg et al. 1989). This analysis was performed for each of the four frequencies where the WM applied input $(1,5,7$ or $9 \mathrm{~Hz})$.

\section{Performance}

The exerted torque, as measured by the torque transducer, as well as the angle, were filtered off-line with a bandpass frequency filter between 0.5 and $30 \mathrm{~Hz}$ to eliminate offset and power supply artefacts (Omlor et al. 2007). In the angle protocol, performance was evaluated with the root-meansquare (RMS) of the difference between the measured torque and the mean torque. These values (one per trial) were added together to calculate the cumulative root mean squared error (cMSE). For the torque protocol, we used the RMS of the difference between measured angle and the mean angle (Patino et al. 2008). One-way Multivariate ANOVA analysis was performed to test for performance differences between controls and patients. To account for intersubject variability and to generate a more symmetrical distribution, cMSE values were logarithmically transformed.

\section{Results}

In all subjects, the WM perturbations generated significant ECR-FCR inter-muscular coherence at the same frequencies as the perturbations (see Fig. 1). To see if the (distally applied) perturbations could alter the low-frequency drive, measured more proximally in the neck, we also examined SC-SCM and SC-DEL IMC.

\section{Frequency shift}

In the angle protocol, SC-SCM IMC (Fig. 2) revealed the low-frequency band between 3 and $7 \mathrm{~Hz}$ in patients 1 through 4. It differed in shape with respect to bandwidth and magnitude. The band was absent in patients 5 and 6 and in controls. The values of the central frequency of the lowfrequency drive, summarized in Table 2, did not shift consistently to higher frequencies with higher-frequency angle perturbations, but rather fluctuated around a common value.

In the torque protocol, SC-SCM IMC revealed a band between 3 and $7 \mathrm{~Hz}$ in patients 2,3 and 4 (shown in Fig. 3.). While it was found in the angle protocol, MD patient 1 did not have the low-frequency drive in this protocol. In addition, in the torque protocol, the central frequency fluctuated rather than increases together with 
Fig. 2 SC-SCM coherence spectra in the angle protocol, for the three different angle perturbations applied by the WM (at $0-4,4-8$ and $8-12 \mathrm{~Hz}$ ) and also for no perturbation (Iso). In each figure, the bottom horizontal line is the $95 \%$ confidence limit; the upper horizontal lines represent the frequencies where the WM applied perturbation. Isometric data from P1 are missing. For each MD patient, spectra are presented (top 6 figures). Averaged spectra are also presented for MD patients and for Control subjects (bottom 2 figures). $S C$ splenius capitis, $S C M$ sternocleido mastoid

Table 2 Frequency of the lowfrequency drive found in SCSCM coherence in MD patients

$M D$ Myoclonus Dystonia patient
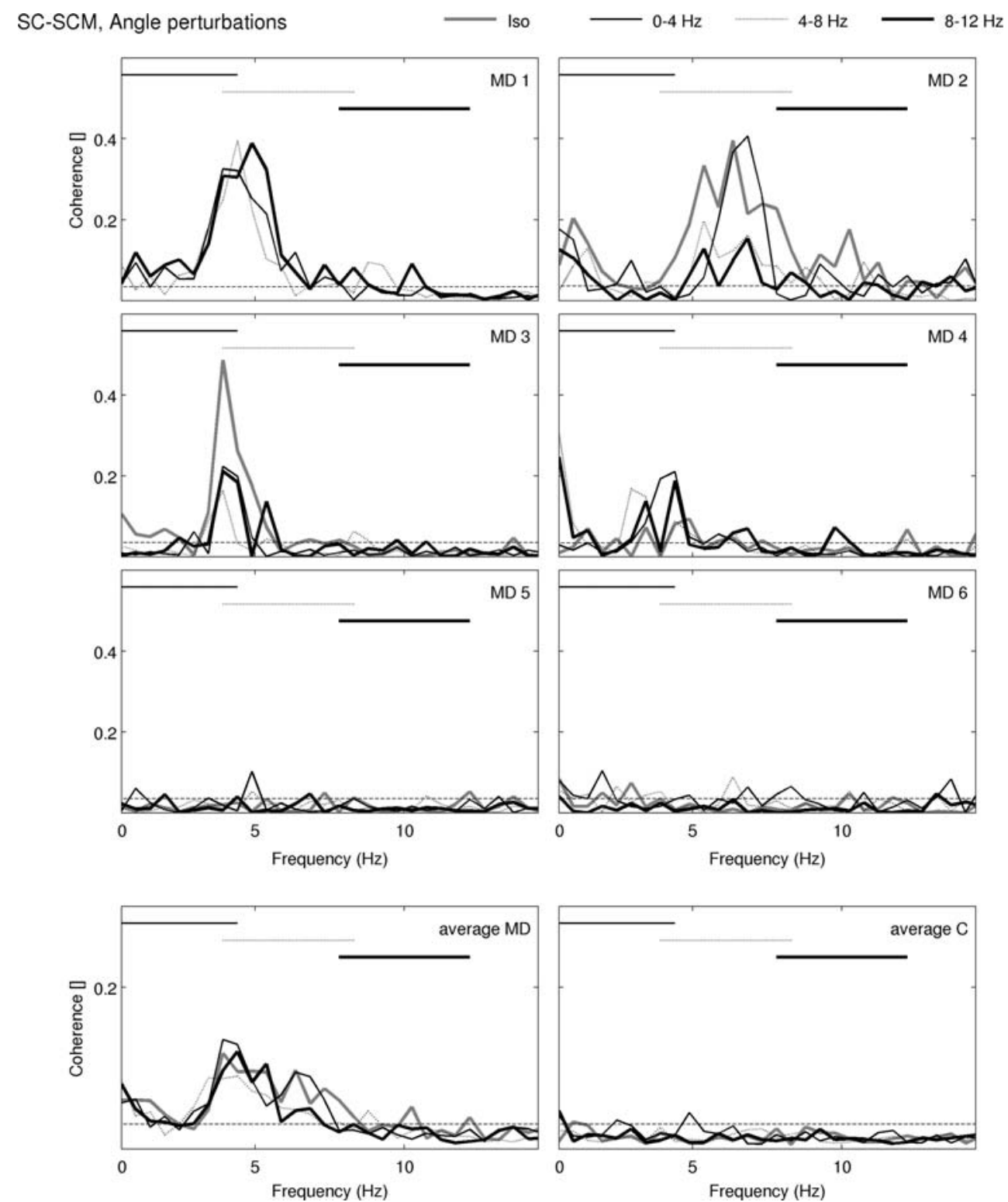

\begin{tabular}{|c|c|c|c|c|c|c|c|c|}
\hline & \multirow[t]{2}{*}{ Isometric } & \multicolumn{3}{|c|}{ Angle perturbations $(\mathrm{Hz})$} & \multicolumn{4}{|c|}{ Torque perturbations $(\mathrm{Hz})$} \\
\hline & & $0-4$ & $4-8$ & $8-12$ & 1 & 5 & 7 & 9 \\
\hline MD 1 & - & 4.37 & 4.26 & 4.31 & - & - & - & - \\
\hline MD 2 & 7.09 & 6.58 & 6.74 & 6.12 & 5.60 & 5.19 & - & 9.26 \\
\hline MD 3 & 4.23 & 4.21 & 3.74 & 4.13 & 4.13 & - & 3.61 & 4.25 \\
\hline MD 4 & 4.66 & 4.03 & 4.75 & 3.30 & 4.49 & 3.67 & - & 5.41 \\
\hline MD 5 & - & - & - & - & - & - & 7.27 & - \\
\hline MD 6 & - & - & - & - & - & 5.83 & - & 9.13 \\
\hline
\end{tabular}

increasing values of the perturbation. There were some exceptions: Although MD patient 2 had a central frequency at $9 \mathrm{~Hz}$ during the $9 \mathrm{~Hz}$ perturbation, the coherence spectra had another significant peak at $5 \mathrm{~Hz}$, close to the central frequency of the low-frequency drive during other pertur- bations. Since it only consisted of a single frequency point, it was not identified as belonging to the low-frequency drive. MD patients 5 and 6 show central frequencies that lie close to the frequency of the perturbation. The average spectra show that MD patients have coherence between 3 
Fig. 3 SC-SCM coherence spectra in the torque protocol, for the four different torque perturbations $(1,5,7$ and $9 \mathrm{~Hz})$. In each figure, the bottom horizontal line is the $95 \%$ confidence limit; the upper vertical lines represent the frequency where the WM applied perturbation. For each MD patient, spectra are presented in the top six figures. Averaged spectra are also presented for MD patients and for Control subjects (bottom 2 figures). $S C$ splenius capitis, $S C M$ sternocleido mastoid
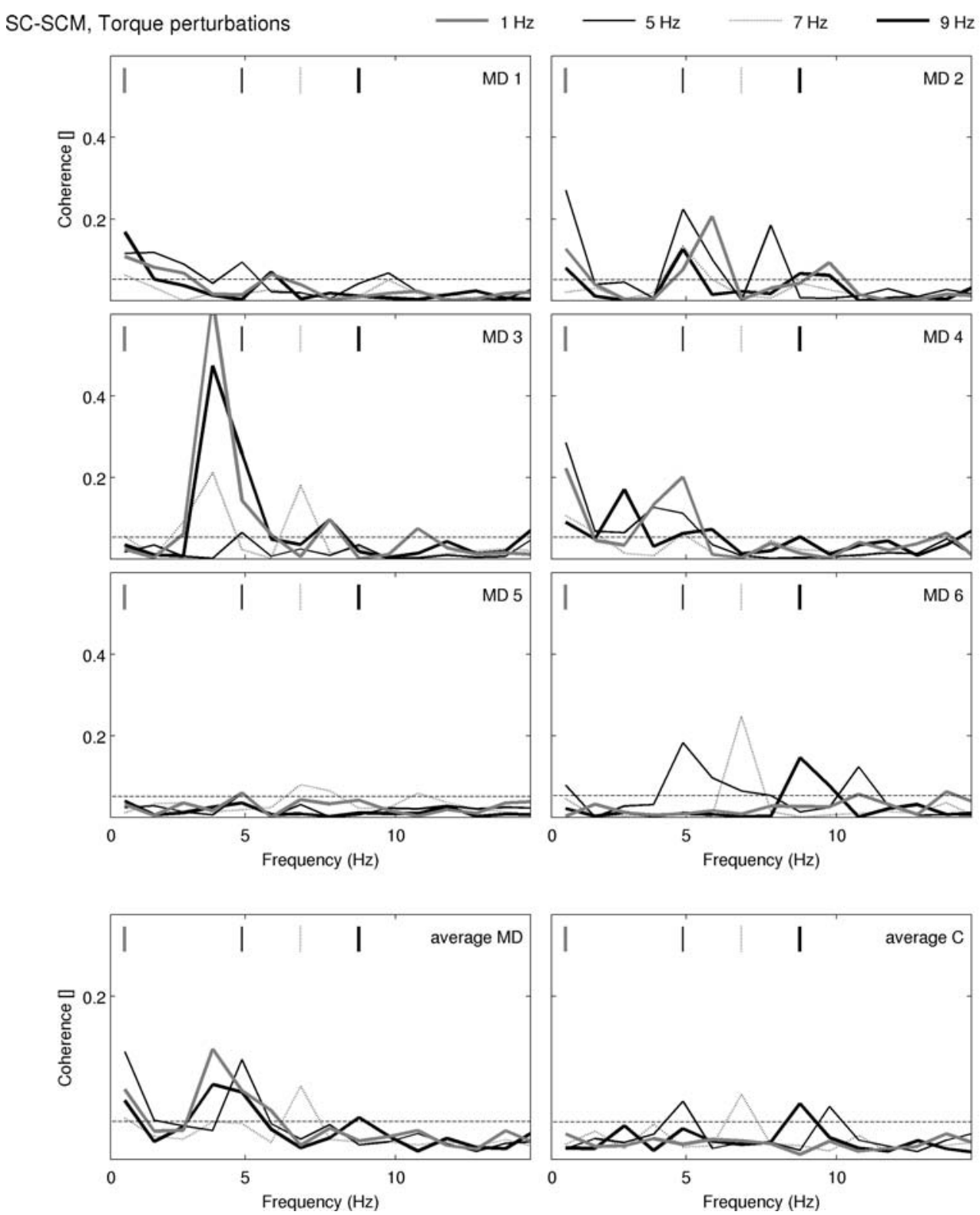

and $7 \mathrm{~Hz}$, and that controls have not. Instead, the average SC-SCM coherence spectra in controls showed elevated peaks at the same frequency as the perturbation, indicative of reflexive feedback.

\section{Reflexive feedback}

To investigate when reflexive feedback occurred in the torque protocol, we analyzed the individual coherence spectra of all subjects for peaks at the perturbation frequency. This was performed for SC-SCM and SC-DEL coherence. Occurrences of reflexive feedback are summarized in Table 3. The average SC-SCM and SC-DEL coherence spectra are shown in Figs. 3 and 4. Table 3 shows that reflexive feedback only occurred for $\mathrm{MD}$ patients 5 and 6 and for controls. Patient 3 had reflexive feedback in SC-SCM coherence during the $7 \mathrm{~Hz}$ perturbation; this was however accompanied by the low-frequency drive with a central frequency at $3.91 \mathrm{~Hz}$ (see Table 2). With this exception, no reflexive feedback occurred in MD patients that had the low-frequency drive. Statistical comparison between the values of the SC-DEL coherence at the input frequencies revealed that on average, patients had significantly lower coherence values than controls in 7 and $9 \mathrm{~Hz}$ perturbations (Wilcoxon rank sum test, $\mathrm{p}<0.008$ and $\mathrm{p}<0.013$, respectively). The average SCDEL and SC-SCM coherence spectra are similar; controls subjects primarily had coherence at the frequency of the WM perturbation and MD patients primarily had coherence near 3-7 Hz. 
Table 3 Perturbations in the torque protocol for which the peak corresponding to reflexive feedback occurred in the intermuscular coherence spectrum

$M D$ Myoclonus Dystonia patient, $C$ Control subject

\begin{tabular}{llllll}
\hline & SC-SCM $(\mathrm{Hz})$ & SC-DEL $(\mathrm{Hz})$ & & SC-SCM $(\mathrm{Hz})$ & SC-DEL $(\mathrm{Hz})$ \\
\hline MD 1 & - & - & C 1 & 7 & 7 \\
MD 2 & - & - & C 2 & - & $5,7,9$ \\
MD 3 & 7 & - & C 3 & 5,7 & $5,7,9$ \\
MD 4 & - & - & C 4 & - & 7 \\
MD 5 & - & 5 & C 5 & - & - \\
MD 6 & 7 & 5,7 & C 6 & 7,9 & $5,7,9$ \\
\hline
\end{tabular}

Table 4 Performance data

\begin{tabular}{llllll}
\hline Angle & MD & Controls & $\begin{array}{l}\text { Torque } \\
(\mathrm{Hz})\end{array}$ & MD & Controls \\
\hline Isometric & $11.9(0.9)$ & $9.8(0.8)$ & 1 & $14.7(0.8)$ & $13.6(0.9)$ \\
$0-4 \mathrm{~Hz}$ & $11.7(1.4)$ & $10.6(0.6)$ & 5 & $15.6(0.8)$ & $15.5(1.1)$ \\
$4-8 \mathrm{~Hz}$ & $11.6(1.3)$ & $10.2(0.4)$ & 7 & $14.9(0.3)$ & $14.9(0.5)$ \\
$8-12 \mathrm{~Hz}$ & $11.7(1.2)$ & $10.7(0.4)$ & 9 & $13.9(0.6)$ & $13.4(0.3)$
\end{tabular}

Performance data and standard deviations of the error across subjects, measured in log cRMSE (cumulative root mean squared error, in the motor: $\log (\mathrm{Nm} / \mathrm{V})$, and sensory: $\log (\mathrm{m} / \mathrm{V})$ protocols). There were no significant performance differences between patients and controls in the motor protocol, but patients performed less well in the sensory protocol. In addition, but only in controls, performance under perturbation conditions was impaired versus the isometric condition. $M D$ Myoclonus Dystonia

displayed neck-C3 CMC. A frequency-shift toward the perturbation frequency was not observed.

\section{Discussion}

Peripheral torque and angle perturbations were applied to MD patients to assess whether sensory input could change the frequency of the dystonic $3-7 \mathrm{~Hz}$ frequency drive in MD. The central findings of this study are as follows. (1) The intermuscular drive was detected in four MD patients in neck-neck ICM. Furthermore, corticomuscular coher-

ficant coherence at 3-7 Hz was also found in C3 CMC in 3 MD patients. No other patients or controls
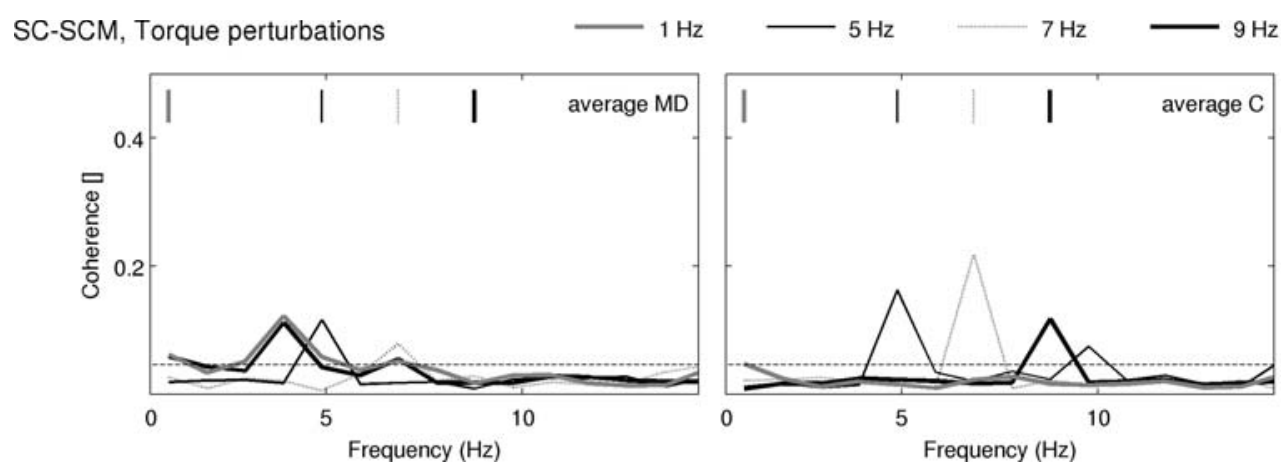

Fig. 4 SC-DEL coherence spectra in the torque protocol, for the four different torque perturbations $(1,5,7$ and $9 \mathrm{~Hz})$. In each figure, the bottom horizontal line is the $95 \%$ confidence limit; the upper vertical lines represent the frequency where the WM applied perturbation. For each

MD patient, spectra are presented in the top 6 figures. Averaged spectra are also presented for MD patients and for Control subjects (bottom 2 figures). $S C$ splenius capitis, $S C M$ sternocleido mastoid 


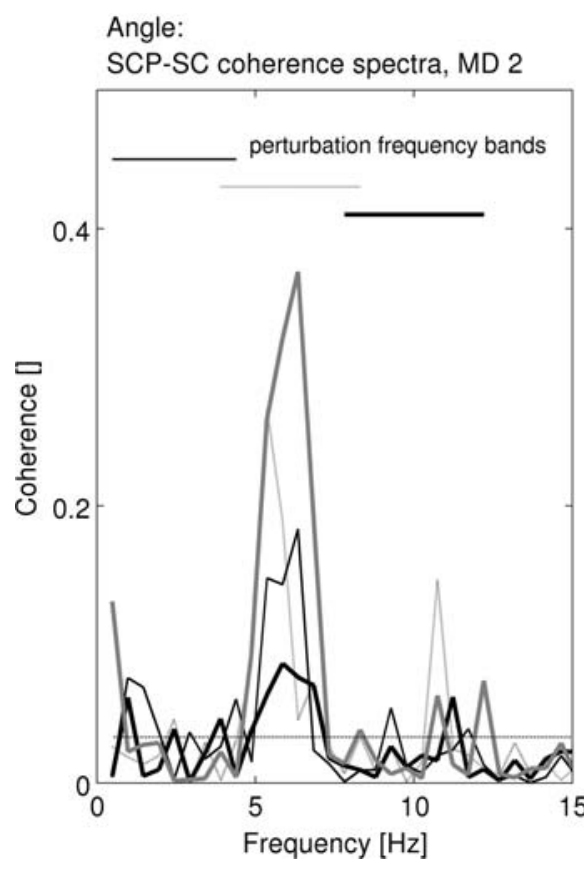

Fig. 5 Corticomuscular coherence. Horizontal dashed lines give the 95\% confidence limits. Left, for the angle protocol, SCP-SC coherence spectra for all conditions are plotted for M-D patient 2. Horizontal lines at the top give the frequency bands of WM input perturbations in the first three conditions; the continuous gray line gives the spectrum for the isometric condition. The 3-7 Hz drive was seen in all four spectra. This remained in the same frequency band although the amplitude

ence in the same frequency band was also detected in three MD patients. (2) Torque or angle perturbations were unable to shift the $3-7 \mathrm{~Hz}$ band of significant coherence to other frequencies. (3) In the torque protocol, patients with the intermuscular low-frequency drive did not have reflexive feedback in SC-SCM and SC-DEL coherence, whereas this was observed in the controls and two patients without the intermuscular 3-7 Hz drive.

\section{Angle protocol}

The frequency of the intermuscular drive in MD patients remained constant despite the wrist perturbations applied: the drive is insensitive to external proprioceptive stimuli from muscle spindles in the arms. The reason why may be as follows: (1) The 3-7 Hz frequency band may not contain all resonating frequencies of the intermuscular drive. It is possible that there are relevant frequencies outside this band and the $3-7 \mathrm{~Hz}$ band is no more than a sub-harmonic residue of intracerebral interactions. (2) The $3-7 \mathrm{~Hz}$ band observed in IMC of dystonic muscles is not the result of resonance between sensory and motor systems that encompasses the sensorimotor cortex and the peripheral muscles, but rather represents a 'resonance' between the motor cortex and other structures including the basal ganglia and the
Torque:

SCP-FCR reflexive feedback, all subjects

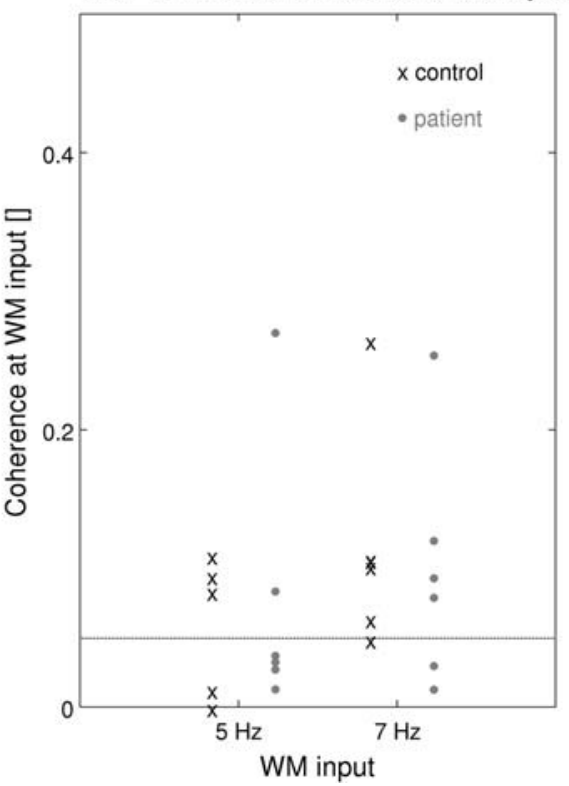

decreased with ascending perturbation frequencies. Right for the torque protocol, SCP-FCR coherence values at $5 \mathrm{~Hz}$ for the $5 \mathrm{~Hz}$ input condition, and $7 \mathrm{~Hz}$ for the $7 \mathrm{~Hz}$ input condition are plotted for patients (black) and controls ( gray). Values did not differ between patients and controls. SCP source derivation the contralateral $\mathrm{CP}(3 / 4)$ location, FCR Flexor Carpis Radiali; SC Splenius Capitis, WM Wrist Manipulator, $M D$ Myoclonus Dystonia

thalamus. A thalamo-cortical oscillatory interaction has been simulated mathematically (Rosenblum and Pikovsky 2004).

Neck-C3 CMC results of MD patient 2 in the angle protocol suggests that increased perturbation frequency causes a dampening effect. However, this could equally well be attributed to vibration-induced pre-synaptic inhibition of SC muscle. This dampening effect could not be seen in patients 2 and 3 .

Torque protocol

FCR-ECR IMC at the perturbation frequencies most likely results from afferent feedback from muscle spindles and Golgi tendon organs. Mono- or bi-synaptic reflex systems in the spinal cord drive the muscle activity of both FCR and ECR muscles with the same frequency as the WM perturbations. This reflexive feedback is picked up with coherence analysis.

The observed SC-DEL reflexive feedback (i.e., IMC at the WM frequency) is more difficult to explain because the proximal muscles are not perturbed in first instance. Furthermore, neurons in the spinal column for controlling these distal muscles are anatomically located at a different height than the neurons responsible for proximal muscles, 
and no information should be exchanged between these groups of neurons at the spinal level. One possible explanation is that intensive co-contraction of the distal muscles increases the overall rigidity of the arm as a whole, and mechanical perturbations are transferred to the shoulder. Another possible explanation is that there are afferent feedback loops in higher areas of the CNS picking up distal sensory information and activate the proximal muscles CMC between hand and $\mathrm{C} 3$ scalp location at WM frequencies demonstrates that proprioceptive information is transmitted to higher brain areas. This would support the notion that the CNS could be involved in the activation of other muscle groups.

The coincidence of the presence of the intermuscular drive with the absence of the perturbation frequencies in shoulder-neck IMC suggests, that the inter-muscular drive in MD patients interferes with normal sensory-motor effects of torque perturbations.

\section{Performance}

There was no significant difference in performance between patients and controls in the torque protocol. This may be due to the generation of co-contraction in this protocol, especially at higher frequencies. Since dystonia in the arm should not impair co-contraction, patient's performance could indeed be comparable to control's performance. In the angle, protocol performance was impaired in MD patients, probably due to the dystonia. Whereas normal subjects maintain a steady torque in the angle protocol using the FCR muscle only, in dystonia the co-contraction of both ECR and FCR probably reduces performance. Another possible explanation is that afferent feedback from muscle spindles is used by the spinal feedback loop in the maintenance of a steady torque. In MD patients, the intermuscular drive then may interfere with normal function of this spinal feedback system.

\section{Corticomuscular coherence}

A previous study has failed to demonstrate significant corticomuscular coherence in MD (Foncke et al. 2007b) but coherence had been found between LFP of the GPi and EMG of the arm muscles in two MD patients, and therefore a cortical relay of the intermuscular drive would seem essential. This may have been due to the limited number of EEG electrodes employed. Therefore, we studied EEG-EMG coherence with a denser packing of electrodes. The findings in $\mathrm{CMC}$ indeed indicate involvement of the sensorimotor cortex in both the intermuscular drive in the sensation of the perturbations of the WM.
Limitations

By focusing on one type of hereditary dystonia, we avoid to a degree heterogeneity in our group of patients. However, even within a genetically homogeneous group, which limb is affected, the severity of dystonia and when dystonia occurs, can vary. We attempted to select patients with dystonia in the neck, but in two cases, the characteristic 3-7 Hz drive was not found. MD patient 1 had the low-frequency drive in the angle protocol, while it was absent in the torque protocol. It could be argued that for calculating the average coherence spectra and the statistical comparisons, we could equally well have chosen to group the first four MD patients into a 'low-frequency drive' group, and the last two MD patients together with the six controls in a "no drive' group. However, since we started out by selecting patients with the DYT-11 mutation, the origin of dystonia is at least identical for every patient.

How sensory-motor system in other types of dystonia such as writer's cramp or facial dystonia would react to the perturbations used in this protocol would probably depend on the type of dystonia and whether the dystonia is present in rest or is induced by action. Probably dystonia in the hands or arms would be more responsive to perturbations that are applied at the arm, as is the case in this study, while cervical dystonia would be less responsive. Cervical dystonia in MD in any case seems to be generated by a more central generator and would therefore be intrinsically unresponsive to outside perturbations. If this is also true for other cases of cervical dystonia would have to be investigated. It could be that if the perturbations were applied at the neck, it would be able to affect the low-frequency drive.

\section{Conclusions}

It seems likely that, even in a situation where the sensorimotor control system is sensitive to external (torque) perturbations, the stronger drive generated by resonance with sub-cortical structures would override normal output. Sensory signals from perturbations would be detected but they would not affect either the resonance between cortical and subcortical structures or the output of the motor system. A recent study reported that information flow in the $3-7 \mathrm{~Hz}$ frequency range is primarily downwards from the CNS to the affected muscles, supporting the idea of a more or less 'independent' dystonic drive (Sharott et al. 2008). Therefore, in MD patients, sensory malfunction may be more secondary to dystonia and the intermuscular drive, rather than a cause.

Acknowledgments This study was supported by the following research grants: NWO-VIDI grant (project 016.056.333). 
Open Access This article is distributed under the terms of the Creative Commons Attribution Noncommercial License which permits any noncommercial use, distribution, and reproduction in any medium, provided the original author(s) and source are credited.

\section{References}

Abbruzzese G, Berardelli A (2003) Sensorimotor integration in movement disorders. Mov Disord 18(3):231-240

Bara-Jimenez W, Catalan MJ et al (1998) Abnormal somatosensory homunculus in dystonia of the hand. Ann Neurol 44(5):828-831

Bloch R (1983) Subtraction of electrocardiographic signal from respiratory electromyogram. J Appl Physiol 55:619-623

Bove M, Brichetto G et al (2004) Neck proprioception and spatial orientation in cervical dystonia. Brain 127:2764-2778

Breakefield XO, Blood AJ et al (2008) The pathophysiological basis of dystonias. Nat Rev Neurosci 9(3):222-234

de Vlugt E, Schouten AC et al (2002) Adaptation of reflexive feedback during arm posture to different environments. Biol Cybern 87(1):10-26

Dresel C, Haslinger B et al (2006) Silent event-related fMRI reveals deficient motor and enhanced somatosensory activation in orofacial dystonia. Brain 129:36-46

Foncke EMJ, Gerrits MCF et al (2006) Distal myoclonus and late onset in a large Dutch family with myoclonus-dystonia. Neurology 67(9):1677-1680

Foncke EMJ, Bour LJ et al (2007a) Local field potentials and oscillatory activity of the internal globus pallidus in myoclonus-dystonia. Mov Disord 22(3):369-376

Foncke EMJ, Bour LJ et al (2007b) Abnormal low frequency drive in myoclonus-dystonia patients correlates with presence of dystonia. Mov Disord 22(9):1299-1307

Gerrits MCF, Foncke EMJ et al (2006) Phenotype-genotype correlation in Dutch patients with myoclonus-dystonia. Neurology 66(5):759-761

Grosse P, Edwards M et al (2004) Patterns of EMG-EMG coherence in limb dystonia. Mov Disord 19(7):758-769

Halliday DM, Rosenberg JR, Amjad AM, Breeze P, Conway BA, Farmer SF (1995) A framework for the analysis of mixed time series/point process data-theory and application to the study of physiological tremor, single motor unit discharges and electromyograms. Prog Biophys Mol Biol 64:237-278

Leis AA, Dimitrijevic MR et al (1992) Modification of cervical dystonia by selective sensory stimulation. J Neurol Sci 110(1-2):79-89

Levine S, Gillen J, Weiser P, Gillen M, Kwatny E (1986) Description and validation of an ECG removal procedure for EMGDI power spectrum analysis. J Appl Physiol 60:1073-1081

Li JY, Cunic DI et al (2008) Electrophysiological features of myoclonus-dystonia. Mov Disord 23(14):2055-2061
McDonnell MN, Thompson PD et al (2007) The effect of cutaneous input on intracortical inhibition in focal task-specific dystonia. Mov Disord 22(9): 1286-1292

Myers LJ, Lowery M et al (2003) Rectification and non-linear preprocessing of EMG signals for cortico-muscular analysis. J Neurosci Methods 124(2):157-165

Naumann M, Magyar-Lehmann S et al (2000) Sensory tricks in cervical dystonia: perceptual dysbalance of parietal cortex modulates frontal motor programming. Ann Neurol 47(3):322-328

Omlor W, Patino L et al (2007) Gamma-range corticomuscular coherence during dynamic force output. Neuroimage 34(3):1191-1198

Patino L, Omlor W et al (2008) Absence of gamma-range corticomuscular coherence during dynamic force in a deafferented patient. J Neurophysiol 99(4):1906-1916

Pintelon R, Schoukens J et al (2003) Uncertainty of transfer function modelling using prior estimated noise models. Automatica 39(10):1721-1733

Rosenberg JR, Amjad AM et al (1989) The Fourier approach to the identification of functional coupling between neuronal spike trains. Prog Biophys Mol Biol 53(1):1-31

Rosenblum M, Pikovsky A (2004) Delayed feedback control of collective synchrony: an approach to suppression of pathological brain rhythms. Phys Rev E 70(4):11

Rosenkranz K, Butler K et al (2008) Sensorimotor reorganization by proprioceptive training in musician's dystonia and writer's cramp. Neurology 70(4):304-315

Sanger TD, Pascual-Leone A, Tarsy D, Schlaug G (2002) Nonlinear sensory cortex response to simultaneous tactile stimuli in writer's cramp. Mov Disord 17:105-111

Schouten AC, de Vlugt E, van Hilten JJB, van der Helm FCT (2006) Design of a torque-controlled manipulator to analyse the admittance of the wrist joint. J Neurosci Methods 154(1-2):134-141

Schouten AC, De Vlugt E et al (2008) Quantifying proprioceptive reflexes during position control of the human arm. IEEE Trans Biomed Eng 55(1):311-321

Sharott A, Grosse P et al (2008) Is the synchronization between pallidal and muscle activity in primary dystonia due to peripheral afferance or a motor drive? Brain 131:473-484

Tarsy D, Simon DK (2006) Current concepts—dystonia. N Engl J Med 355(8):818-829

Tijssen MAJ, Munchau A et al (2002) Descending control of muscles in patients with cervical dystonia. Mov Disord 17(3):493-500

van der Helm FCT, Schouten AC et al (2002) Identification of intrinsic and reflexive components of human arm dynamics during postural control. J Neurosci Methods 119(1):1-14

Vitek JL (2002) Pathophysiology of dystonia: a neuronal model. Mov Disord 17:S49-S62

Yanagisawa N, Goto A (1971) Dystonia musculorum deformans. Analysis with electromyography. J Neurol Sci 13(1):39-65 